\title{
Effects of X-Rays on Perovskite Solar Cells
}

\author{
Michael E. Stuckelberger, ${ }^{*, \dagger, \perp}$ Tara Nietzold, ${ }^{\ddagger}$ Bradley M. West, ${ }^{\ddagger}$ Yanqi Luo, \\ Xueying Li, " Jérémie Werner, ${ }^{\S}$ Björn Niesen, ${ }^{\S}$ Christophe Ballif, ${ }^{\S}$ Volker \\ Rose,"\# David P. Fenning, and Mariana I. Bertoni ${ }^{\ddagger}$ \\ $\dagger$ †eutsches Elektronen-Synchrotron, Photon Science, Notkestr. 85, 22607 Hamburg, \\ Germany \\ $\ddagger$ Arizona State University (ASU), Ira A. Fulton Schools of Engineering, School of \\ Electrical, Computer and Energy Engineering (ECEE), Defect Lab, 551 E. Tylor Mall, \\ Tempe, AZ 85287, United States of America \\ ฯUniversity of California San Diego, Department of Nanoengineering, La Jolla, CA 92093, \\ United States of America \\ $\S$ Ecole Polytechnique Fédérale de Lausanne (EPFL), Institute of Microengineering (IMT), \\ Photovoltaics and Thin Film Electronics Laboratory (PVLAB), Rue de la Maladière 71, \\ 2000 Neuchâtel, Switzerland \\ \|Argonne National Laboratory (ANL), Advanced Photon Source (APS), 9700 S. Cass \\ Avenue, Lemont, IL 60439, United States of America \\ $\perp$ Arizona State University (ASU), Ira A. Fulton Schools of Engineering, School of \\ Electrical, Computer and Energy Engineering (ECEE), Defect Lab, 551 E. Tylor Mall, \\ Tempe, AZ 85287, United States of America \\ \#Argonne National Laboratory (ANL), Center for Nanoscale Materials (CNM), 9700 \\ S. Cass Avenue, Lemont, IL 60439, United States of America \\ E-mail: michael.stuckelberger@desy.de
}

Abstract - Synchrotron micro- and nanoprobe beamlines have demonstrated great potential to advance photovoltaic devices. Most importantly, their small $\mathrm{X}$-ray spotsize has enabled the direct correlation of electrical performance with elemental composition at sub-grain resolution for a variety of polycrystalline solar cells. Whereas the bulk of most inorganic semiconductors is stable under the high X-ray flux of focused Xray beams, semiconductors with organic components are prone to a variety of degradation mechanisms. This is particularly critical to evaluate for the emerging organometal halide perovskite solar cells. Here, we investigate the effects of hard X-rays on the nanoscale per- formance and elemental distribution of these solar cells. We show that their composition does not change during common operando and in-situ measurements at synchrotron nanoprobes. However, we found a significant X-ray induced electronic degradation of solar cells with methylammonium lead iodide absorbers. Time- and dose-dependent measurements unveiled two characteristic degradation time constants on the order of 12 and $200 \mathrm{~s}$ that are independent of the X-ray flux. Based on heat and dose simulations, we attribute the fast decay to the dose-driven creation of recombination centers, while the slow decay is compatible with the observation of compositional changes. Finally, we detail how 
degradation-induced measurement artifacts can be outrun and showcase the high correlation of the X-ray beam induced current with the iodine and lead distribution.

\section{Introduction}

The advent of correlative X-ray microscopy ${ }^{1}$ in photovoltaic research is largely based on the co-development of brilliant hard-X-ray synchrotron micro- and nanoprobe beamlines at third-generation synchrotrons and of experimental techniques such as X-ray beam induced current $(\mathrm{XBIC})^{1-4}$ and voltage (XBIV). ${ }^{5-7}$ These operando measurement techniques are conceptually related to electron- (EBIC) and laser-beam induced current (LBIC), and give access to the charge collection efficiency in the absorber layer, providing the high penetration depth of LBIC at the high spatial resolution of EBIC. Enabled by the great penetration depth in low- $Z$ materials and by the long working distance, hard X-rays are intrinsically suited to serve as probe beams for in-situ measurements of complete solar cell stacks under various conditions. $^{6,7}$

In the framework of multimodal scanning X-ray microscopy, ${ }^{8}$ the combination of XBIC/XBIV with X-ray fluorescence (XRF) and nano-diffraction measurements has turned out to be particularly powerful for the point-by-point correlation of the electrical performance with the elemental composition and strain in thin-film solar cells with compound polycrystalline absorber layers such as $\mathrm{CuIn}_{1-x} \mathrm{Ga}_{x} \mathrm{Se}_{2},{ }^{6,9,10} \mathrm{CdTe},{ }^{11,12}$ or methyl-ammonium lead iodide (MAPI, $\left.\mathrm{CH}_{3} \mathrm{NH}_{3} \mathrm{PbI}_{3}\right) \cdot{ }^{13-17}$

The record efficiency of perovskite solar cells (PSCs) with an organometal halide absorber has been increasing at an unprecedented rate, being after a few years of development already beyond the record efficiency of other more established polycrystalline thin-film solar cells. ${ }^{18}$ This justifies the tremendous research efforts, although upscaling challenges and degradation have hindered large-scale im- plementation of PSCs so far. Perovskite solar cells suffer from a variety of degradation mechanisms caused by humidity, light, atmosphere, and combinations thereof. ${ }^{19-23}$ Furthermore, damage of operational PSCs and their absorber layer has been reported from electron and X-ray beams. ${ }^{13,24,25}$ For advanced characterization at the nanoscale - most importantly, to study degradation mechanisms operando and in-situ - it is therefore critical to understand and quantify the probe-induced modifications.

\section{Methods}

\section{Solar cell fabrication}

To study the X-ray beam induced damage of PSCs, we have synthesized perovskite solar cells on glass substrates with fluorinated tin oxide (FTO) that serves as front contact during standard solar cell operation. A compact and a mesoporous layer of titanium dioxide was deposited onto the FTO by sputtering and spin coating, respectively. The archetypal MAPI was spin coated as absorber layer on the $\mathrm{TiO}_{2}$ scaffold following the procedure described elsewhere. ${ }^{26}$ As hole-transport layer, spiro-MeOTAD was spin coated on the absorber, and gold was evaporated as back contact. The solar cell synthesis is detailed elsewhere in greater detail, ${ }^{27}$ and the samplespecific details are provided in the supporting information (SI) in Tab. S4.

\section{$\mathrm{XRF}$ and XBIC measurements}

The experiments were performed at the nanoprobe beamline 26-ID-C ${ }^{28}$ at Argonne National Laboratory. The angle between the XRF detector and incident X-ray beam is fixed to $90^{\circ}$. The angle between the normal to the sample surface and the incident X-ray beam was $15^{\circ}$, optimized to reduce self-absorption effects in the XRF signal while minimizing the footprint of the X-ray beam. A zone plate focused the coherent X-ray beam at $9 \mathrm{keV}$ to $30-40 \mathrm{~nm}$ (FWHM), resulting in a photon flux on the order of $2.6 \times 10^{8}$ photons $/ \mathrm{s}$. 
The strong X-ray absorption in glass did not allow measuring the XBIC signal with the glass facing the incident X-ray beam, which would correspond to standard solar cell operation. Therefore, the X-ray beam entered the solar cell through the rear contact (p-type) that was grounded to avoid measurement artifacts as discussed elsewhere. ${ }^{1}$ The low X-ray absorptance in MAPI leads to a flat charge generation profile throughout the solar cell thickness that is comparable to the illumination with red light and depends only marginally on the illumination side. To reduce the X-ray beam intensity, aluminum filters of 114 and $343 \mu \mathrm{m}$ were used with an X-ray transmittance of 35.3 and $4.4 \%$, respectively. All data shown here are normalized to the X-ray flux assessed by an up-stream ion chamber to mitigate artifacts from the decaying electron current in the storage ring.

The careful reader will note that the molar ratio between iodine and lead differs significantly from the nominal stoichiometric ratio of 3 that is expected for the perovskite crystals. This difference is predominantly caused by measurement artifacts: self-absorption of fluorescence photons, errors of thin-film standard calibration, and limited comparability of the spectrum fitting for $\mathrm{Pb}_{\mathrm{M}}$ and $\mathrm{I}_{\mathrm{L}}$ peaks cause an apparently off-stoichiometric ratio between the lead and iodine distributions, in this case an underestimation of lead. For further discussion of these effects on the elemental quantification in thin-film devices such as perovskite solar cells, we refer to the literature. ${ }^{7,17,29,30}$ Note that these errors in the absolute quantification of elements cancel out in relative evaluation. Consequently, these effects to not affect the outcome of this study, as all conclusions will be drawn from a relative comparison of elemental concentrations as a function of time and space.

\section{Simulations}

For the quantification of the dose distribution, we have performed numeric simulations of the X-ray photon/solar-cell interaction using a personalized version of PENCYL that is included in the PENELOPE software pack- age. $^{31,32}$ The dose profiles shown in Fig. S6 \& S7 have been obtained with $10^{8}$ simulated incident photons. The simulation parameters were the same as the experimental parameters (9 keV incident-photon energy, layer stack with the thicknesses and compositions as detailed in Tab. S4), except for the beam diameter and the beam/sample-surface angle that were $0 \mathrm{~nm}$ and $90^{\circ}$, respectively.

For the evaluation of the temperature distribution, heat transfer simulations were performed in COMSOL Multiphysics $\mathbb{R}$ software. ${ }^{33}$ To simulate the heat flux coming from the X-ray beam absorbed in the sample, we assumed a line source at the origin of uniform magnitude (in $\mathrm{W} / \mathrm{m}$ ) through the thickness of each layer. The magnitude of the line source in each layer was taken from the absorbed energy per X-ray photon in each layer calculated in the PENELOPE dose simulations at a flux of $2.6 \times 10^{8}$ photons $/ \mathrm{s}$ per the beamline operating specification. X-ray measurements were taken in vacuum with the sample integrated atop a thick glass substrate, such that heat transfer from the sample is poor. To establish a worst-case heating scenario, we assumed no heat transfer across the upper or lower boundaries of the sample, meaning that we overestimated the temperature rise in the absorber. Further details about the simulations are given in the SI.

\section{Results}

\section{Effects of X-rays on absorber com- position}

First, we shall investigate the impact of X-ray irradiation on the compositional distribution in the solar cell absorber. ${ }^{1}$ Therefore, we have subsequently measured a solar cell area 5 times as shown in Fig. 1 and published elsewhere; ${ }^{14}$ a $6^{\text {th }}$ map has been taken slightly larger. Comparing the pristine vs. re-measured areas by eye, we barely note X-ray beam induced modifications, despite of the uncommonly long dwell

\footnotetext{
${ }^{1}$ Note that these measurements based on hard X-rays are not sensitive to compositional variations of organic absorber components.
} 


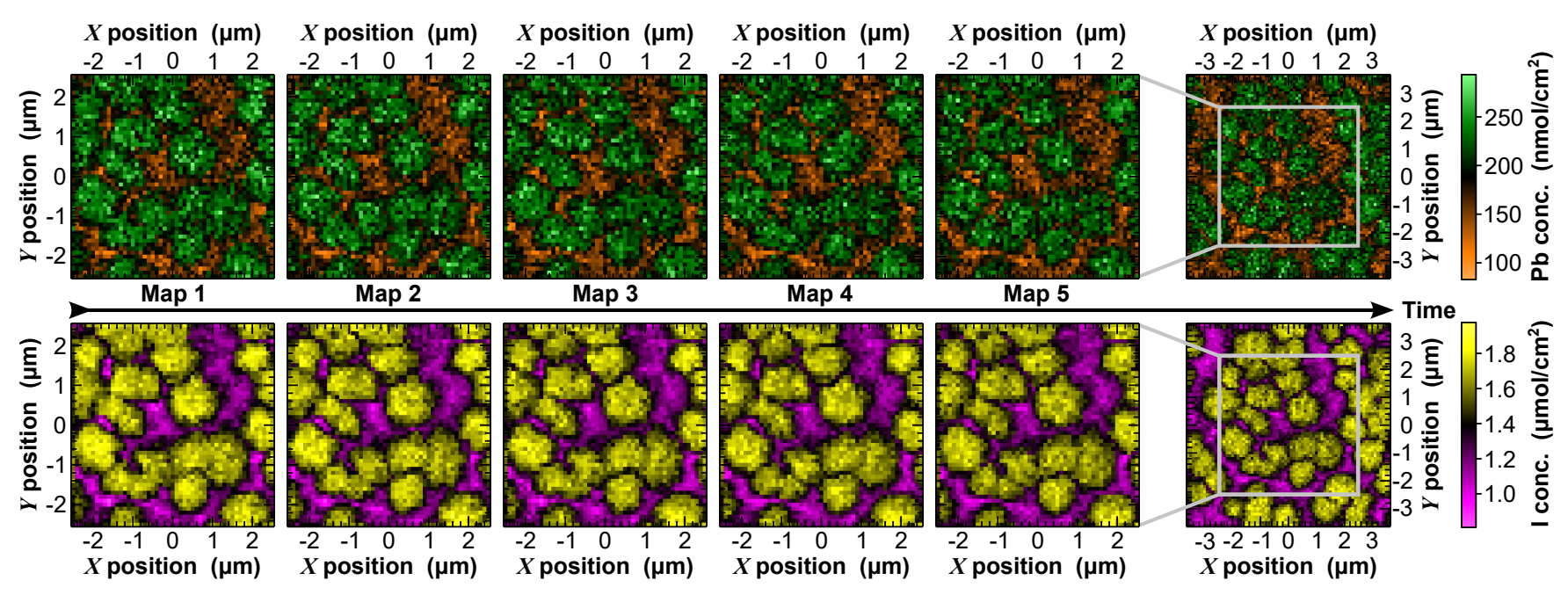

Figure 1: Repeated X-ray fluorescence measurement of a perovskite solar cell area to investigate X-ray beam induced changes of the compositional distribution. Top: lead distribution. Bottom: iodine concentration. All maps were taken with $100 \mathrm{~nm} \times 100 \mathrm{~nm}$ pixel size and $1 \mathrm{~s}$ dwell time. Reproduced with permission from Adv. Funct. Mater. 2018, 1706995. (c) 2018 WILEY-VCH Verlag GmbH \& Co. KGaA, Weinheim.

time of $1 \mathrm{~s}$ per pixel. As detailed in the SI, we conclude that compositional variations of the absorber layer are not relevant within commonly used dwell times.

\section{Effects of X-rays on charge collec- tion}

Figure 2 illustrates the problem of X-ray beam induced electrical degradation of PSCs with a MAPI absorber, when the solar cells are naively measured at X-ray nanoprobes under typical conditions used for other thin-film solar cell measurements (here: $0.5 \mathrm{~s}$ dwell time and $100 \mathrm{~nm}$ step size). Figure 2(a) shows the iodine distribution resulting from the XRF measurement, where no degradation is apparent. In contrast, the simultaneously taken XBIC measurement shown in Fig. 2(b) clearly shows a strong signal at the beginning of the scan at coordinates $(X, Y)=(-2 \mu \mathrm{m},-2 \mu \mathrm{m})$ that decreases to $50 \%$ within the first few lines. The strong electronic degradation masks any underlying structure in the XBIC signal. The spatial confinement of the degradation is visible in Fig. 2(c), for which a quick overview scan was taken with larger step sizes, outrunning the degradation induced by the second scan.

By comparison of Fig. 2(c) with the $4 \mu \mathrm{m} \times$ $4 \mu \mathrm{m}$ sized measurement of Fig. 2(b), we note

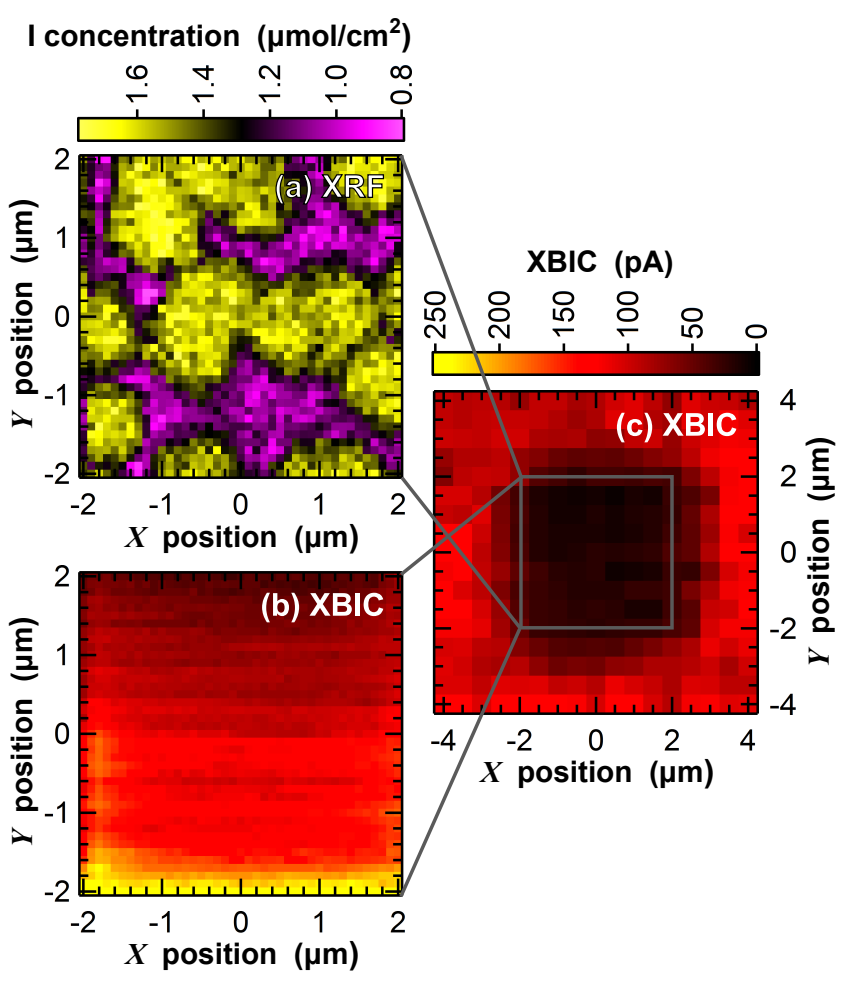

Figure 2: Apparent X-ray beam induced degradation of a perovskite solar cell in a synchrotron $X$-ray nanoprobe. Left: un-impacted iodine distribution from $X$-ray fluorescence measurements (a), and the degrading X-ray beam induced current $(X B I C)(b)$. The measurement started from the left bottom and continued in horizontal lines to the upper right corner. Right: XBIC map of a larger area (c) including the area that was mapped with high resolution. The color scales of both XBIC measurements are equal. 
first that the "electronic crater" is slightly larger than the originally scanned area. Second, we notice that the solar cell performance has decreased even few micrometers away from the "electronic crater": the XBIC does not reach beyond $50 \%$ of the maximum XBIC signal of the pristine sample. Yet, it is noticeable that the X-ray beam induced damage is laterally contained. A few dozen micrometers away from the irradiated spot, the solar cell properties are unaffected: the XBIC signal reached values that were comparable to the first XBIC measurements of the pristine solar cell. Furthermore, the open-circuit voltage of the solar cell - evaluated upon illumination of the entire solar cell inside the vacuum chamber by visible light - did not change within the measurement precision.

On one hand, this means that no significant shunts between the two electrodes are created by the X-rays. On the other hand, it indicates that the defects lead to spread-out recombination and act as a sink for the electron-hole pairs that are generated within the diffusion length. Alternative explanations for the apparently spread-out degradation that will be discussed later include beam-induced heating effects and degradation from the tails in the $\mathrm{X}$ ray profile.

For the assessment of the degradation kinetics of the electrical performance, we have measured the XBIC signal during continuous focused irradiation of 3 distinct spots with different X-ray intensities corresponding to 100 , 35.3 , and $4.4 \%$ of the maximum focused flux of $2.6 \times 10^{8}$ photons $/ \mathrm{s}$. The pristine spots were first illuminated during $11 \mathrm{~min}$ - the integrated time of a fast XBIC scan-followed by a minute with shutter closed and another minute with shutter open again. The results are shown in Fig. 3. In all three cases, the signal intensity decreased within 11 minutes to about $50 \%$ of the respective initial state as shown in the inset. Note that the degradation kicks in only after a few seconds, which leaves enough time for a quick XBIC measurement before the scan continues to the next pristine spot.

As expected, the XBIC signal decay appears faster at elevated X-ray intensities; quantita-

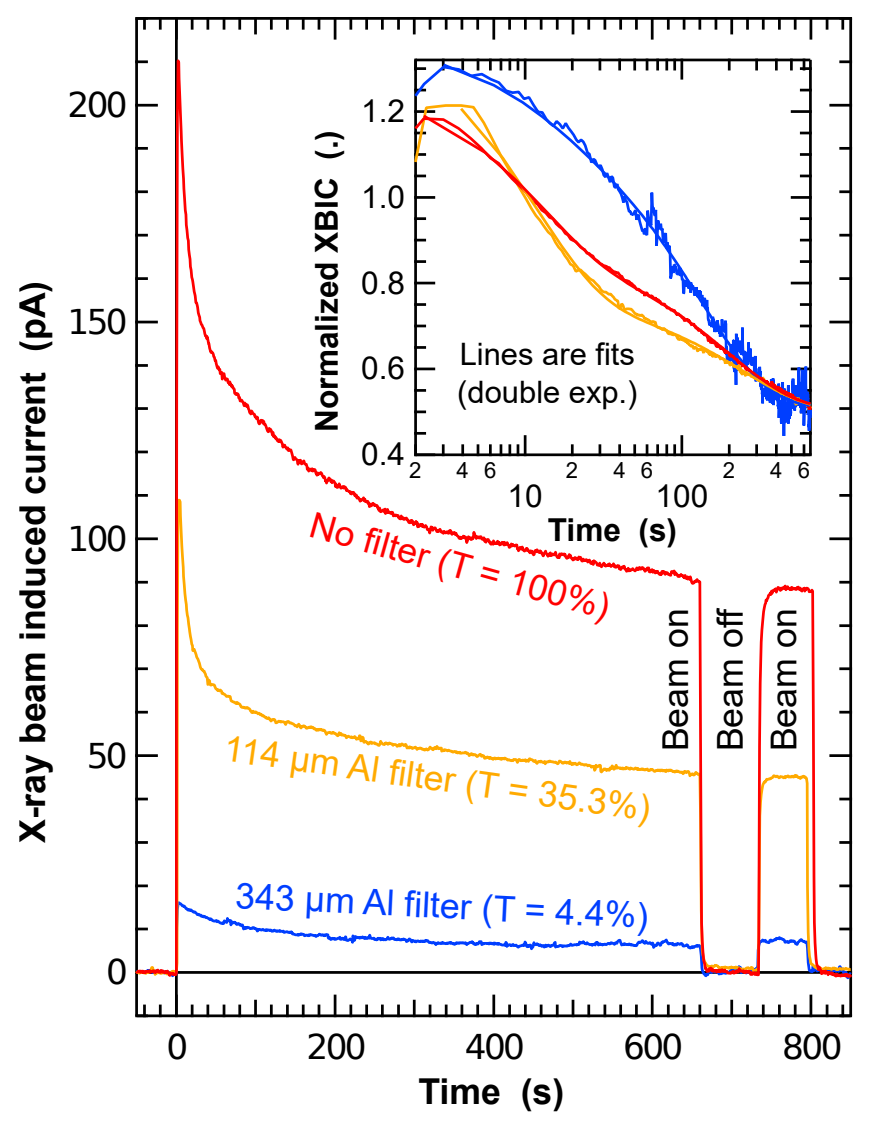

Figure 3: Decay of the XBIC signal at 3 pristine spots upon irradiation with a focused $X$-ray beam that was attenuated with Al filters to 100, 35.3, and 4.4\% of the full incident beam. The inset shows the $X B I C$ signal after normalization to the filter transmittance and fitted to double exponential functions. The resulting fit parameters are given in Tab. S2.

tive results from the fit with double exponential functions are given in Tab. S2. A closer look at the decay rates unveils a fast and slow decay with characteristic decay times that are on the order of 12 and $200 \mathrm{~s}$, respectively. This suggests two distinct degradation mechanisms such as the generation of electronic defects (recombination-active states in the bandgap) followed by the chemical re-arrangement (details are provided in the SI). Note that the fitting unveils similar values of both time constants independent of the flux. However, the low-flux decay shows significantly smaller amplitude of the fast, and larger amplitude of the slow component. As a result, the effective decay appears slower at short time scales for the low flux.

For the assessment of the lateral expansion 
of the degradation effect, we have performed quick XBIC scans across the damaged areas (see Fig. 4), in both $X$ and $Y$ directions in the sample surface plane. In order to reduce the effect of spatial non-uniformities that are responsible for XBIC variations next to the "electronic crater," the XBIC signal is normalized to the medium XBIC signal of the plateau next to it. In agreement with the previous measurements, we observe a decrease of the XBIC signal to $50 \%$ around $(X, Y)=(0,0)$ where the beam had been parked for a total of $12 \mathrm{~min}$. More remarkable is the lateral dimension of the damage: with increasing irradiation intensity, the diameter of the damaged area increases up to $4 \mu \mathrm{m}$, i.e. two orders of magnitude larger than the FWHM beam size. Note that the "electronic craters" are considerably larger than the highly localized "compositional craters" that occur upon extended irradiation (details are provided in the SI, see Fig. S4).
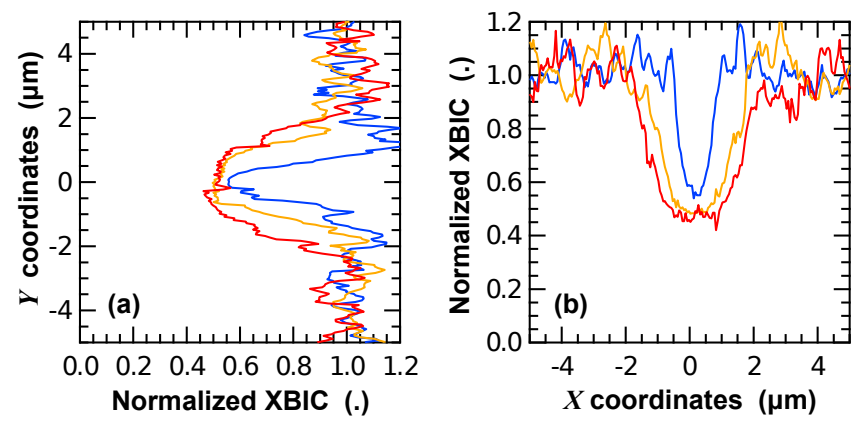

Figure 4: (a) Vertical and (b) horizontal extent of the $X$-ray beam induced damage, evaluated by quick XBIC scans across the points irradiated for Fig. 3.

\section{Causes for degradation}

We considered four possible causes for the observed X-ray beam induced degradation: (1) temperature-induced decomposition from local heating by the X-ray beam; (2) X-ray beam induced ablation; (3) compositional changes such as ion diffusion; (4) dose-related creation of electronic defect states by the ionizing X-ray irradiation.

(1) We have simulated the X-ray beam induced heating in a simplified perovskite solar cell stack as shown in Fig. S5. Although we were overestimating the temperature increase by limiting the sample volume and prohibiting heat transfer at boundaries, the simulations indicate no temperature increase beyond a few $\mathrm{K}$, neither at short- $(10 \mu \mathrm{s})$ nor long-time scales (100s). Furthermore, the high thermal conductivity of the sample leads to fast thermal equilibration, which is not compatible with the spatially confined degradation. Therefore, we exclude X-ray beam induced temperature increase as the dominant cause of the X-ray beam induced decay of the electrical signal.

(2) Cold X-ray beam induced ablation can occur in a similar way as laser ablation. Athermal ablation requires pulse duration in the femtosecond range, which is not compatible with the pulses that are about 50 ps long at the synchrotron. Furthermore, ablation is expected to significantly reduce the area density of the ablated material (at least gold as the uppermost material), and the XBIC signal is expected to vanish upon ablation. Neither of these ablation signatures is compatible with the measurements. Therefore, we exclude ablation as mechanism responsible for the observed XBIC degradation.

(3) As detailed in the SI, extended exposure to focused X-ray beams leads to a slight reduction of the local concentration of lead and iodine. Overall, the time constants of the elemental-concentration decay are comparable to the slow time constant of the XBIC signal decay, indicating that compositional variations may be responsible for the slow XBIC decay. These results are in agreement with the effects of substoichiometry observed earlier. ${ }^{13,14}$ The larger degradation amplitude and lateral extension of the XBIC signal can be explained by the large diffusion length of charge carriers in the absorber.

However, the results do not support the hypothesis of compositional variations causing the fast XBIC decay at the time scale of seconds.

(4) For an estimation of the X-ray dose that is absorbed in the perovskite solar cell during XBIC measurements, we have simulated the energy density being deposited by $10^{8}$ photons. We have found that the interaction volume, within which $68 \%$ of the dose in the absorber is deposited, spans a diameter of about $200 \mathrm{~nm}$, 


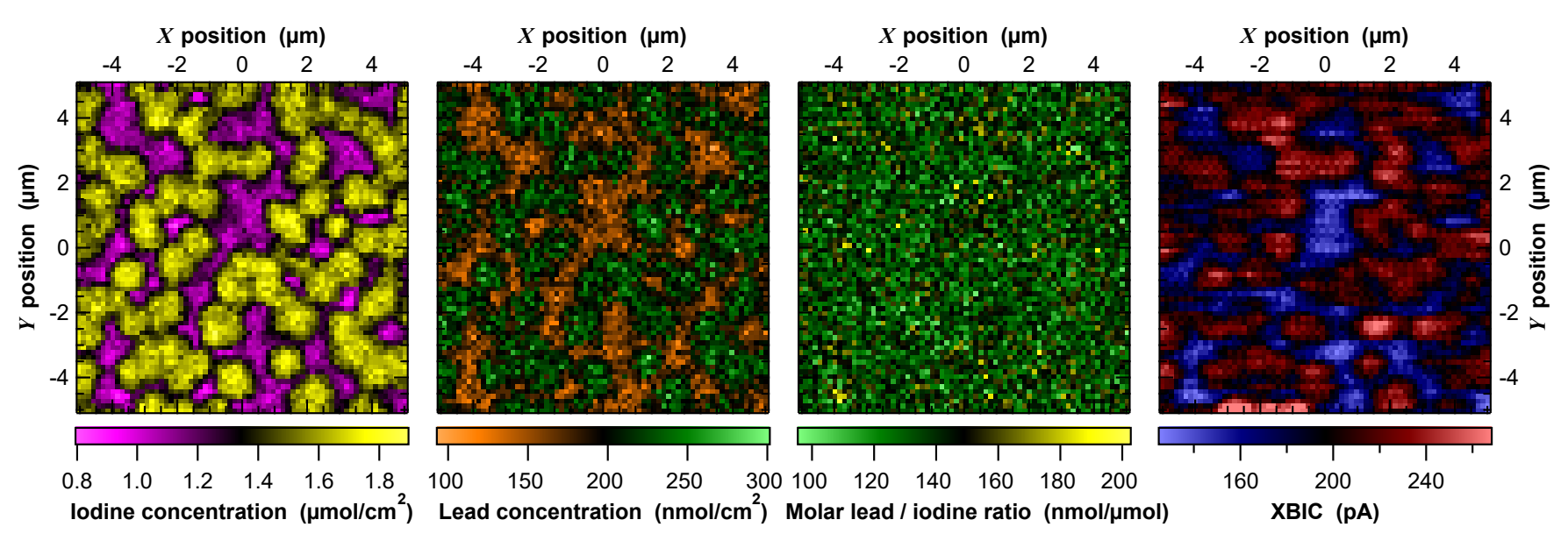

Figure 5: For a comparison of the elemental distribution from XRF measurements with the nanoscale performance from XBIC measurements in the trade-off between signal-to-noise ratio and X-ray beam induced degradation, the XBIC measurement was taken first with attenuated X-ray beam (filter transmittance: $35.3 \%)$ and short dwell time (0.5 s) followed by an XRF measurement with unattenuated beam and $1 \mathrm{~s}$ dwell time.

which gives an idea of the intrinsic limitation of the spatial resolution of such XBIC measurements as detailed elsewhere. ${ }^{7,17}$

We note that pixels in the beginning of a scan have absorbed significantly less dose prior to the XBIC evaluation there compared to subsequently measured pixels. Figure S7 guides through the process of convoluting the X-ray beam size with the dose distribution from a pencil-like X-ray beam. These simulations are in full agreement with the rapid decrease of the XBIC signal observed experimentally (see Fig. 2(b)), and with the XRF maps that do not suffer from degradation to the same extent. These simulations suggest that the large difference of the interaction radii for XBIC and XRF measurements, being on the order of $100 \mathrm{~nm}^{2}$ and $15 \mathrm{~nm}$, respectively, may further widen the lateral dispersion of XBIC degradation compared to elemental concentration, in addition to the broadening of the XBIC degradation by the charge-carrier diffusion.

These results imply that the electronic degradation may be induced by the deposited dose leading to enhanced recombination, whereas compositional degradation occurs only locally and at a slower time scale.

\footnotetext{
${ }^{2}$ The interaction radius of $100 \mathrm{~nm}$ from high-energy secondary photons and electrons is only a lower limit and does not take into account the diffusion length of the charge carriers that may be larger.
}

\section{Outrunning degradation artifacts during XBIC measurements}

Mapping the electrical performance of a degrading sample is often challenging, particularly if the recombination-enhanced area is larger than the beam footprint and step size in combined XBIC/XRF measurements. Nevertheless, there are ways to perform high-quality XBIC/XRF measurements that are hardly affected by the detrimental measurement-induced degradation effects. In the following, we will shortly discuss best-practice approaches that may be combined for greatest signal-to-noise in both XBIC and XRF measurements.

\section{Optimize distinct scans}

Combined XBIC/XRF measurements are a special case of multi-modal measurements with simultaneous acquisition of different modalities that require a compromise of scan settings that are not compatible for all modalities. ${ }^{8}$ In this case, taking subsequent scans of the same area as shown in our previous work ${ }^{13}$ may lead to the best results: a first scan is optimized for XBIC measurements with short dwell times and attenuated beam to limit beam-induced degradation. A second scan covering the same area can then be optimized for high sensitivity and spatial resolution of XRF measurements. Due to drift and 

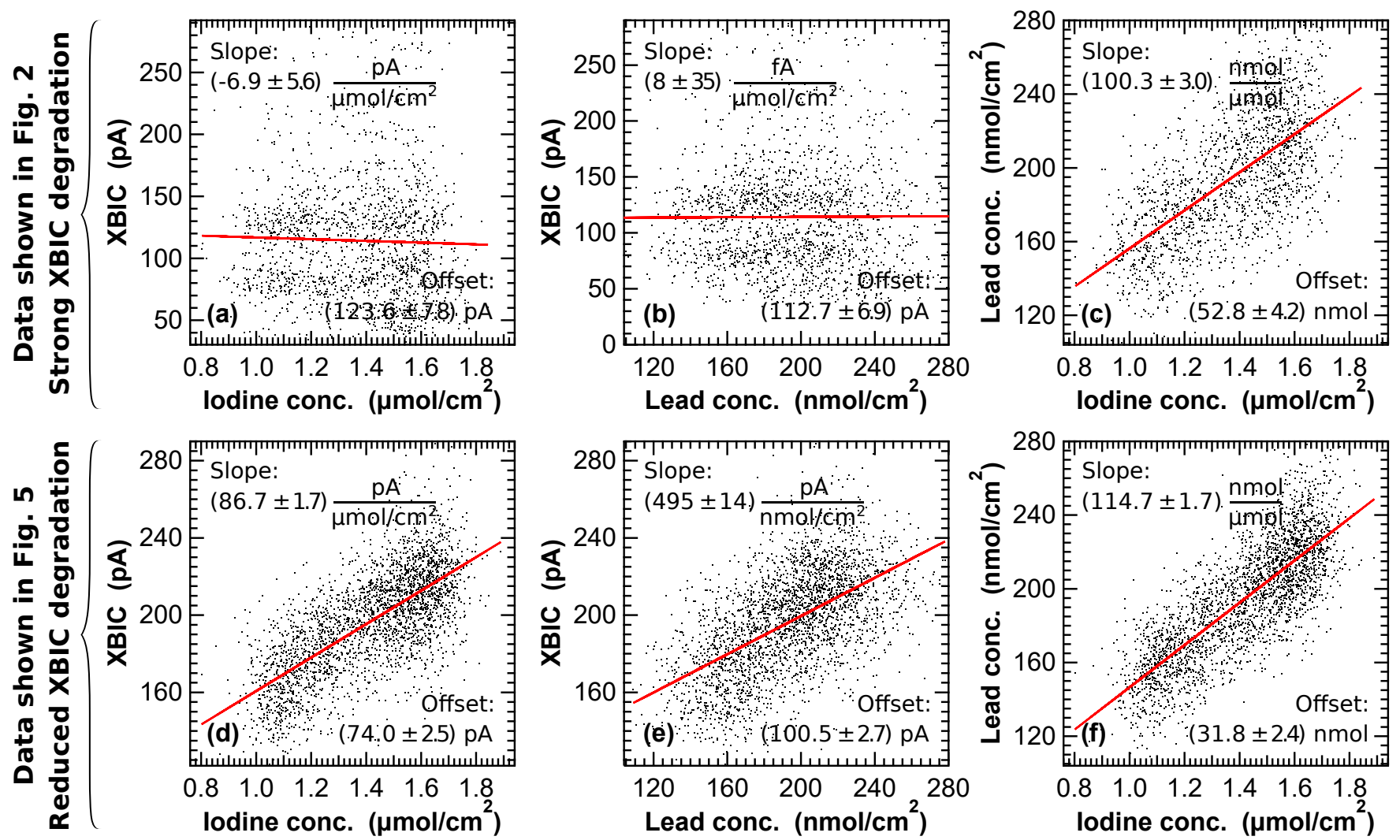

Figure 6: Based on the XBIC and XRF maps shown in Fig. 2855, the scatter plots show the correlation between the electrical performance, the lead concentration, and the iodine concentration. Each dot represent one pixel, and the red lines are the result of linear fits. The insets indicate the slope and offset with the standard deviation.

lack of reproducibility of scan positions that are inevitable at the nanoscale, the XBIC and XRF measurements will require alignment e.g. by image registration. ${ }^{34}$

\section{Decrease measurement time}

Given that the signal-to-noise ratio in XBIC measurements is rather limited by currents induced by the environment than by statistics, the integration time can be even below $1 \mathrm{~ms}$ if the response chain of the solar cell/amplifier/data acquisition system is fast enough. However, scanning speed may be limiting, in particular if no continuous 'fly-scan mode $^{35}$ or fast shutter are available, such that the sample degrades during the settling time prior to the measurement at each scan position. In this case, the scan step size may be increased to match the diameter of the interaction volume that ultimately limits the spatial resolution and can be larger than the probe diameter at X-ray nano- and microprobe endstations. This approach has been demonstrated earlier. ${ }^{1}$

\section{Use lock-in amplification}

By using lock-in amplification, the signal-tonoise ratio of XBIC measurements can be increased by orders of magnitude. ${ }^{4,7,36}$ This allows the X-ray beam to be attenuated without sacrificing the signal-to-noise ratio, ${ }^{3}$ which leads to a significant reduction of beam damage.

\section{Correlation of XBIC and XRF}

Here, we showcase an example of dealing with the trade-off between high-resolution XRF measurements and minimizing electrical degradation during XBIC measurements. First, the XBIC measurement was taken with the nanofo-

\footnotetext{
${ }^{3}$ Varying the beam intensity leads to different charge-injection levels in the solar cell, thus the chargecollection and recombination mechanisms may change.
} 
cused X-ray beam attenuated to $35.3 \%$ and $0.5 \mathrm{~s}$ dwell time. The scan speed was limited by the motor settling time of $0.3 \mathrm{~s}$ at each scan step. Lacking of fly-scan mode and a fast shutter, the high-resolution XBIC scan still suffered from degradation artifacts with the signature of the inverted pattern of Fig. S7; these artifacts were corrected as described elsewhere. ${ }^{13}$ While such a correction is clearly not ideal for a quantitative analysis, it still allows to analyze the overall $\mathrm{XBIC}$ distribution of degrading samples.

Second, the XRF measurement was taken with unattenuated beam and $1 \mathrm{~s}$ dwell time. The resulting XRF spectra were fitted using MAPS ${ }^{37,38}$ to extract the molar iodine and lead area concentrations from the $\mathrm{I}_{\mathrm{L}}$ and $\mathrm{Pb}_{\mathrm{M}}$ lines, respectively. From the iodine and lead distribution, the molar lead-to-iodine ratio was determined. Figure 5 shows the results of these subsequent scans that were optimized for XBIC and XRF measurements, respectively. In contrast to earlier studies, where areas of particularly high lead-to-iodine concentration indicated the presence of a $\mathrm{PbI}_{2}$ phase that was correlated with low XBIC signal, ${ }^{1,13}$ such features are not clearly visible here, which is typical for a higher quality of the solar cell.

Maps as in Fig. 5 lay the foundations for a correlative analysis between electrical performance and elemental distribution as shown in Fig. 6 . Here, the iodine and lead concentrations are correlated with each other and the XBIC signal, and linear fits were applied to all scatter plots resulting from the point-by-point correlations. This analysis is shown in Fig. $6(\mathrm{a}-\mathrm{c})$ for the measurement with degradation artifacts (data from Fig. 2), and in Fig. 6(d-f) for the optimized measurement with fewer degradation artifacts (data from Fig. 5).

As expected, Fig. 6(c\&f) show a high correlation between the lead and iodine concentration that were measured simultaneously and are dominated by topological variations. However, the decaying XBIC signal does not correlate with the elemental distribution, as unveiled by the large uncertainty of the fitted slope in Fig. 6(a-b).

In contrast, a strong correlation was found between the XBIC signal and the iodine and lead concentrations of the optimized measurements (see Fig. 6(d-e)) as indicated by the small standard deviation of the slope. The clear correlation between $\mathrm{XBIC}, \mathrm{Pb}$, and $\mathrm{I}$ shows that measurement-induced degradation of the film no longer dominates the XBIC signal and instead, chemical inferences can be made.

\section{Conclusions}

Our approach correlating the X-ray beam induced photocurrent with the elemental distribution at the nanoscale shines light on the relation between performance and composition that is often driven by smallest defects. Although XBIC measurements locally damage perovskite solar cells irreversibly, we have demonstrated ways how to acquire high-quality XBIC data despite of the presence of degradation effects. Furthermore, we have quantified the degradation kinetics and run simulations to elucidate the reasons behind. As a result, we could exclude that the degradation is caused by temperature increase. Instead, the results suggest that the degradation of the photocurrent is governed by the dose deposited in the absorber layer across the interaction volume.

This detailed study of experimental parameters for successful measurements of the degradation-sensitive perovskite solar cells will open the door for the in-situ and operando characterization of perovskite solar cells. This is of particular interest in view of the nanoprobe endstations at fourth-generation synchrotrons ${ }^{39-42}$ that will enable experiments with $2-3$ orders of magnitude higher focused $\mathrm{X}$-ray flux.

There is no doubt that higher flux will lead to more pronounced dose-induced sample degradation. However, based on this study we may speculate that a dose-independent, slower, degradation component-related for example to diffusion coefficients - could be outrun by fast measurements at a higher dose rate.

Ultimately, such correlative X-ray microscopy experiments will foster the understanding of the relationship between composition and charge collection at the nanoscale and accelerate the 
development of superior solar cell chemistries and fabrication approaches of perovskite solar cells.

\section{Supporting Information}

SI 1: Further time-dependent XBIC and XRF measurements to support the kinetics measurements

SI 2: Heat transfer simulation

SI 3: Simulation of the X-ray interaction volume and the resulting dose upon scanning $\mathrm{X}$ ray microscopy measurements

Acknowledgement We greatly acknowledge Dr. Martin Holt (ANL) and Geneviève Hall (ASU) for fruitful discussions, and Chris Roehrig (ANL) for technical support. We acknowledge funding from the U.S. Department of Energy under contract DEEE0005848. Use of the Advanced Photon Source, and the Center for Nanoscale Materials, Office of Science user facilities, were supported by the U.S. Department of Energy, Office of Science, Office of Basic Energy Sciences, under Contract No. DE-AC02-06CH11357. This material is based upon work at UC San Diego supported by the National Science Foundation under Grant No. DMR-1848371 and the California Energy Commission Advance Breakthrough award EPC-16-050. The research leading to these results has received funding from Deutsches Elektronen-Synchrotron DESY.

\section{References}

(1) Stuckelberger, M.; West, B.; Nietzold, T.; Lai, B.; Maser, J. M.; Rose, V.; Bertoni, M. I. Review: Engineering solar cells based on correlative X-ray microscopy. Journal of Materials Research 2017, 32, 1825-1854.

(2) Hieslmair, H.; Istratov, A. A.; Sachdeva, R.; Weber, E. R. New synchrotron-radiation based technique to study localized defects in silicon: "EBIC" with X-ray excitation. $10^{\text {th }}$ Workshop on
Crystalline Silicon Solar Cell Materials and Processes 2010, 162-165.

(3) Vyvenko, O. F.; Buonassisi, T.; Istratov, A. A.; Hieslmair, H.; Thompson, A. C.; Schindler, R.; Weber, E. R. Xray beam induced current-a synchrotron radiation based technique for the in situ analysis of recombination properties and chemical nature of metal clusters in silicon. Journal of Applied Physics 2002, 91, 3614-3617.

(4) Ossig, C.; Nietzold, T.; West, B. M.; Bertoni, M. I.; Falkenberg, G.; Schroer, C. G.; Stuckelberger, M. E. $\mathrm{X}$-ray beam induced current measurements for multi-modal X-ray microscopy of solar cells. Journal of Visual Experiments 2019, e60001.

(5) Stuckelberger, M.; Nietzold, T.; West, B. M.; Lai, B.; Maser, J.; Rose, V.; Bertoni, M. I. X-Ray beam induced voltage: A novel technique for electrical nanocharacterization of solar cells. Proc. Photovoltaic Specialist Conference (PVSC) 2017, 2179-2184.

(6) Stuckelberger, M.; Nietzold, T.; West, B. M.; Farshchi, R.; Poplavskyy, D.; Bailey, J.; Lai, B.; Maser, J.; Bertoni, M. I. How does CIGS performance depend on temperature at the microscale? IEEE Journal of Photovoltaics 2018, 8, 278287.

(7) Stuckelberger, M. E.; Nietzold, T.; West, B. M.; Farshchi, R.; Poplavskyy, D.; Bailey, J.; Lai, J. M., B. Maser; Bertoni, M. I. Defect activation and annihilation in CIGS solar cells: an operando x-ray microscopy study. Journal of Physics: Energy 2020, 2, 025001.

(8) Stuckelberger, M. E. Multimodal scanning X-ray microscopy at nanoprobe endstations of fourth-generation synchrotrons. Spectroscopy 2019, 34, 42-44. 
(9) West, B.; Stuckelberger, M.; Guthrey, H.; Chen, L.; Lai, B.; Maser, J.; Rose, V.; Shafarman, W.; Al-Jassim, M.; Bertoni, M. I. Grain engineering: How nanoscale inhomogeneities can control charge collection in solar cells. Nano Energy 2017, 32, 488493.

(10) Ulvestad, A.; Hruszkewycz, S. O.; Holt, M. V.; Hill, M. O.; CalvoAlmazán, I.; Maddali, S.; Huang, X.; Yan, H.; Nazaretski, E.; Chu, Y. S. et al. Multimodal X-ray imaging of grain-level properties and performance in a polycrystalline solar cell. Journal of Synchrotron Radiation 2019, 26, 1316-1321.

(11) Stuckelberger, M.; West, B.; Husein, S.; Guthrey, H.; Al-Jassim, M.; Chakraborty, R.; Buonassisi, T.; Maser, J. M.; Lai, B.; Stripe, B. et al. Latest developments in the x-ray based characterization of thin-film solar cells. Proc. Photovoltaic Specialist Conference (PVSC) 2015, 1-6.

(12) Calvo-Almazán, I.; Ulvestad, A. P.; Colegrove, E.; Ablekim, T.; Holt, M. V.; Hill, M. O.; Maddali, S.; Lauhon, L. J.; Bertoni, M. I.; Huang, X. et al. Strain Mapping of CdTe Grains in Photovoltaic Devices. IEEE Journal of Photovoltaics 2019, 9, 1790-1799.

(13) Stuckelberger, M.; Nietzold, T.; Hall, G. N.; West, B.; Werner, J.; Niesen, B.; Ballif, C.; Rose, V.; Fenning, D. P.; Bertoni, M. I. Charge collection in hybrid perovskite solar cells: relation to the nanoscale elemental distribution. IEEE Journal of Photovoltaics 2017, 7, 590-597.

(14) Luo, Y.; Aharon, S.; Stuckelberger, M.; Magana, E.; Lai, B.; Bertoni, M. I.; Etgar, L.; Fenning, D. P. The relationship between chemical flexibility and nanoscale charge collection in hybrid halide perovskites. Advanced Functional Materials 2018, 1706995.
(15) Li, X.; Luo, Y.; Holt, M. V.; Cai, Z.; Fenning, D. P. Residual nanoscale strain in cesium lead bromide perovskite reduces stability and shifts local luminescence. Chemistry of Materials 2019, 31, 2778-2785.

(16) Correa-Baena, J.-P.; Luo, Y.; Brenner, T. M.; Snaider, J.; Sun, S.; Li, X.; Jensen, M. A.; Hartono, N. T. P.; Nienhaus, L.; Wieghold, S. et al. Homogenized halides and alkali cation segregation in alloyed organic-inorganic perovskites. Science 2019, 363, 627-631.

(17) Kodur, M.; Kumar, R. E.; Luo, Y.; Cakan, D. N.; Li, X.; Stuckelberger, M. E.; Fenning, D. P. X-ray Microscopy of Halide Perovskites: Techniques, Applications, and Prospects. Advanced Energy Materials 2020, 1903170.

(18) Green, M. A.; Dunlop, E. D.; HohlEbinger, J.; Yoshita, M.; Kopidakis, N.; Ho-Baillie, A. W. Solar cell efficiency tables (version 55). Progress in Photovoltaics: Research and Applications 2020, 28, 3-15.

(19) Leguy, A. M. A.; Hu, Y.; CampoyQuiles, M.; Isabel Alonso, M.; Weber, O. J.; Azarhoosh, P.; van Schilfgaarde, M.; Weller, M. T.; Bein, T.; Nelson, J. et al. Reversible hydration of $\mathrm{CH}_{3} \mathrm{NH}_{3} \mathrm{PbI}_{3}$ in films, single crystals, and solar cells. Chemistry of Materials 2015, 27, 3397-3407.

(20) Hall, G. N.; Stuckelberger, M.; Nietzold, T.; Hartman, J.; Park, J.-S.; Werner, J.; Niesen, B.; Cummings, M. L.; Rose, V.; Ballif, C. et al. The role of water in the reversible optoelectronic degradation of hybrid perovskites at low pressure. The Journal of Physical Chemistry C 2017, 121, 25659-25665.

(21) Leijtens, T.; Eperon, G. E.; Noel, N. K.; Habisreutinger, S. N.; Petrozza, A.; Snaith, H. J. Stability of metal halide perovskite solar cells. Advanced Energy $\mathrm{Ma}$ terials 2015, 1500963. 
(22) Snaith, H. J.; Hacke, P. Enabling reliability assessments of pre-commercial perovskite photovoltaics with lessons learned from industrial standards. Nature Energy 2018, 3, 459-465.

(23) Rong, Y.; Hu, Y.; Mei, A.; Tan, H.; Saidaminov, M. I.; Seok, S. I.; McGehee, M. D.; Sargent, E. H.; Han, H. Challenges for commercializing perovskite solar cells. Science 2018, 361, eaat8235.

(24) Yuan, H.; Debroye, E.; Janssen, K.; Naiki, H.; Steuwe, C.; Lu, G.; Moris, M.; Orgiu, E.; Uji-i, H.; De Schryver, F. et al. Degradation of methylammonium lead iodide perovskite structures through light and electron beam driven ion migration. The Journal of Physical Chemistry Letters 2016, 7, 561-566.

(25) Rothmann, M. U.; Li, W.; Zhu, Y.; Liu, A.; Ku, Z.; Bach, U.; Etheridge, J.; Cheng, Y.-B. Structural and chemical changes to $\mathrm{CH}_{3} \mathrm{NH}_{3} \mathrm{PbI}_{3}$ Induced by electron and gallium ion beams. Advanced Materials 2018, 30, 1800629.

(26) Jeon, N. J.; Noh, J. H.; Kim, Y. C.; Yang, W. S.; Ryu, S.; Seok, S. I. Solvent engineering for high-performance inorganicorganic hybrid perovskite solar cells. $\mathrm{Na}$ ture Materials 2014, 13, 897-903.

(27) Werner, J.; Dubuis, G.; Walter, A.; Löper, P.; Moon, S.-J.; Nicolay, S.; Morales-Masis, M.; De Wolf, S.; Niesen, B.; Ballif, C. Sputtered rear electrode with broadband transparency for perovskite solar cells. Solar Energy Materials \&6 Solar Cells 2015, 141, 407-413.

(28) Winarski, R. P.; Holt, M. V.; Rose, V.; Fuesz, P.; Carbaugh, D.; Benson, C.; Shu, D.; Kline, D.; Stephenson, G. B.; McNulty, I. et al. A hard X-ray nanoprobe beamline for nanoscale microscopy. Journal of Synchrotron Radiation 2012, 19, 1056-1060.
(29) West, B.; Stuckelberger, M.; Jeffries, A.; Gangam, S.; Lai, B.; Stripe, B.; Maser, J.; Rose, V.; Vogt, S.; Bertoni, M. I. X-ray fluorescence at nanoscale resolution for multicomponent layered structures: a solar cell case study. Journal of Synchrotron Radiation 2017, 24, 288-295.

(30) Ziska, C.; Ossig, C.; Pyrlik, N.; Carron, R.; Avancini, E.; Fevola, G.; Kolditz, A.; Siebels, J.; Kipp, T.; Cai, Z. et al. Quantifying the elemental distribution in solar cells from X-ray fluorescence measurements with multiple detector modules. Proc. Photovoltaic Specialist Conference (PVSC) 2020,

(31) Salvat, F.; Fernández-Varea, J. M.; Sempau, J. PENELOPE-2006: A code system for Monte Carlo simulation of electron and photon transport. Workshop proceedings. 2006 .

(32) Salvat, F.; Fernancez-Varea, J. M.; Acosta, E.; Sempau, J. PENELOPE2014, a code system for Monte Carlo simulation of electron and photon transport. Available at: http://www.oecd-nea.org/ tools/abstract/detail/nea-1525, (accessed February 15, 2020).

(33) COMSOL Multiphysics@. Stockholm, Sweden. Available at: http: //www.comsol.com, (accessed February 15, 2020).

(34) Pyrlik, N.; Ossig, C.; Ziska, C.; Fevola, G.; Garrevoet, J.; Falkenberg, G.; Schropp, A.; Stuckelberger, M. E. Image registration in multi-modal scanning microscopy: a solar cell case study. Proc. Photovoltaic Specialist Conference (PVSC) 2020,

(35) Morishige, A. E.; Laine, H. S.; Looney, E. E.; Jensen, M. A.; Vogt, S.; Li, J. B.; Lai, B.; Savin, H.; Buonassisi, T. Increased throughput and sensitivity of synchrotron-based characterization for photovoltaic materials. IEEE Journal of Photovoltaics 2017, 7, 763-771. 
(36) Stuckelberger, M.; Nietzold, T.; West, B. M.; Walker, T.; Ossig, C.; Wittwer, F.; Deng, J.; Maser, J. M.; Lai, B.; Cai, Z. et al. Challenges and opportunities with highly brilliant Xray sources for multi-modal in-situ and operando characterization of solar cells. Microscopy and Microanalysis 2018, 24 (Suppl. 2), 434-435.

(37) Vogt, S. MAPS: A set of software tools for analysis and visualization of 3D X-ray fluorescence data sets. Journale de Physique IV 2003, 104, 635-638.

(38) Nietzold, T.; West, B. M.; Stuckelberger, M.; Lai, B.; Vogt, S.; Bertoni, M. I. Quantifying X-ray fluorescence data using MAPS. Journal of Visual Experiments 2018, 132.

(39) Hettel, R. The Advanced Photon Source Upgrade Plan Approved. Synchrotron Radiation News 2019, 32, 34-35.

(40) Schroer, C. G.; Agapov, I.; Brefeld, W.; Brinkmann, R.; Chae, Y.-C.; Chao, H.-C.; Eriksson, M.; Keil, J.; Nuel Gavaldà, X.; Röhlsberger, R. et al. PETRA IV: the ultralow-emittance source project at DESY. Journal of Synchrotron Radiation 2018, 25, 1277-1290.

(41) Raimondi, P. ESRF-EBS: The Extremely Brilliant Source Project. Synchrotron Radiation News 2016, 29, 8-14.

(42) Tavares, P. F.; Al-Dmour, E.; Andersson, A.; Cullinan, F.; Jensen, B. N.; Olsson, D.; Olsson, D. K.; Sjöström, M.; Tarawneh, H.; Thorin, S. et al. Commissioning and first-year operational results of the MAX IV $3 \mathrm{GeV}$ ring. Journal of Synchrotron Radiation 2018, 25, 12911316.

(43) Haynes, W. M. CRC Handbook of Chemistry and Physics. 2016; http:// hbcponline.com, last accessed February 15, 2020 .
(44) Onoda-Yamamuro, N.; Matsuo, T.; Suga, H. Calorimetric and IR spectroscopic studies of phase transitions in methylammonium trihalogenoplumbates (II). Journal of Physics and Chemistry of Solids 1990, 51, 1383-1395.

(45) Guo, Z.; Yoon, S. J.; Manser, J. S.; Kamat, P. V.; Luo, T. Structural Phase- and Degradation-Dependent Thermal Conductivity of CH3NH3PbI3 Perovskite Thin Films. The Journal of Physical Chemistry C 2016, 120, 6394-6401.

(46) Hata, T.; Giorgi, G.; Yamashita, K. The Effects of the Organic-Inorganic Interactions on the Thermal Transport Properties of $\mathrm{CH}_{3} \mathrm{NH}_{3} \mathrm{PbI}_{3}$. Nano Letters 2016, 16, 2749-2753.

(47) Smith, D. S.; Grandjean, S.; Absi, J.; Kadiebu, S.; Fayette, S. Grain-boundary thermal resistance in polycrystalline oxides: alumina, tin oxide, and magnesia. High Temperatures-High Pressures 2003, 35, 93-100.

(48) Schott, Data sheet AF 32 thin glass. http: //www.schott.com, last accessed February 15, 2020.

(49) Solaronix, 2016; http://www . solaronix. com, last accessed February 15, 2020.

(50) Stoumpos, C. C.; Malliakas, C. D.; Kanatzidis, M. G. Semiconducting tin and lead iodide perovskites with organic cations: phase transitions, high mobilities, and near-infrared photoluminescent properties. Inorganic Chemistry 2013, 52, 9019-9038.

(51) Peuchert, U.; Brix, P. Alkali-free aluminoborosilicate glasses, and uses thereof. 2007. 


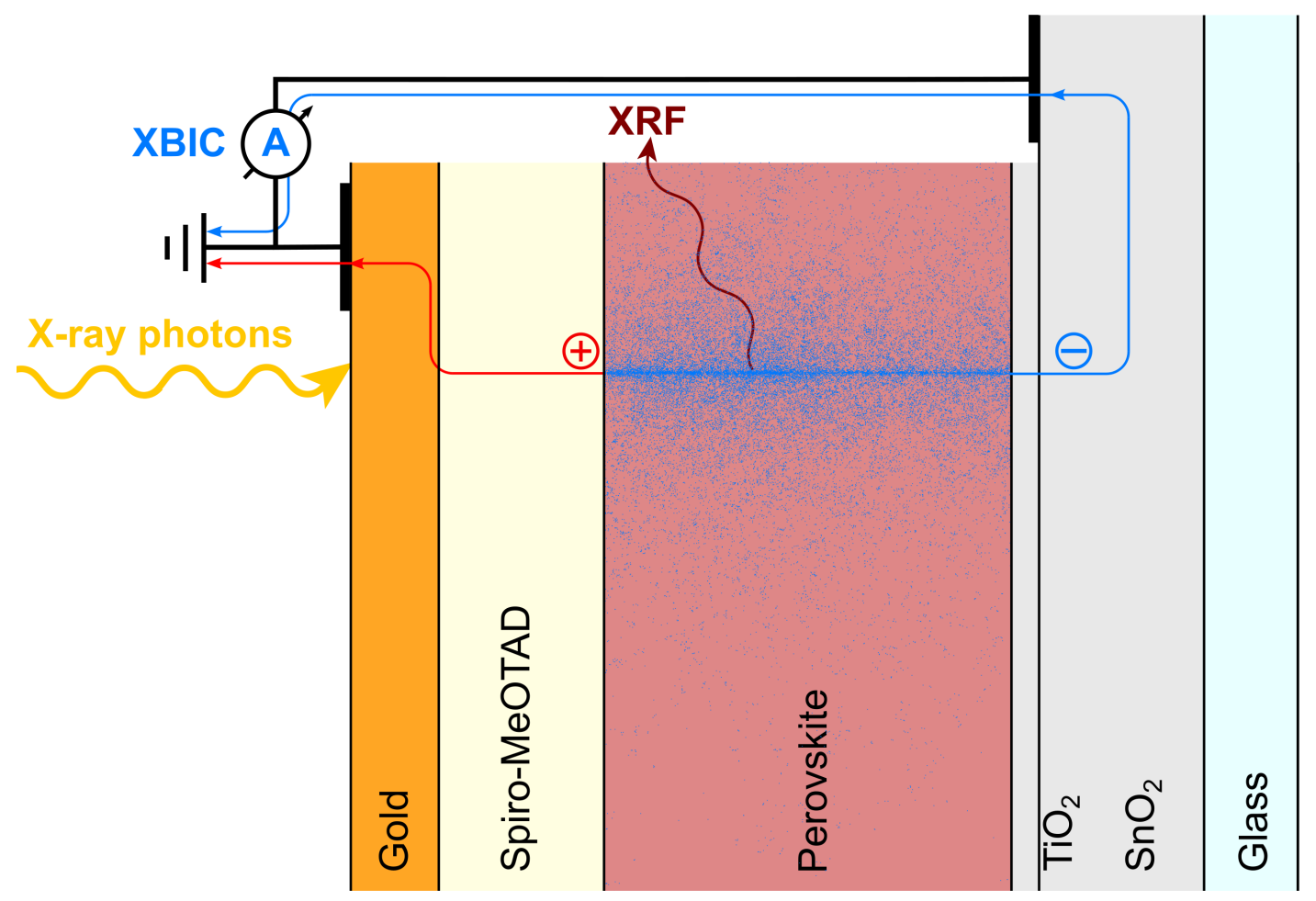

Figure for TOC 